\title{
ON A VARIATION OF A CONGRUENCE OF SUBBARAO
}

\author{
ANDREJ DUJELLA ${ }^{\otimes}$ and FLORIAN LUCA \\ (Received 18 December 2010; accepted 1 February 2012; first published online 4 February 2013) \\ Communicated by F. Pappalardi \\ Dedicated to the memory of Alf van der Poorten

\begin{abstract}
We study positive integers $n$ such that $n \phi(n) \equiv 2 \bmod \sigma(n)$, where $\phi(n)$ and $\sigma(n)$ are the Euler function and the sum of divisors function of the positive integer $n$, respectively. We give a general ineffective result showing that there are only finitely many such $n$ whose prime factors belong to a fixed finite set. When this finite set consists only of the two primes 2 and 3 we use continued fractions to find all such positive integers $n$.
\end{abstract}

2010 Mathematics subject classification: primary 11A25; secondary 11A55.

Keywords and phrases: congruences, arithmetic functions, continued fractions.

\section{Introduction}

We write $\phi(n)$ and $\sigma(n)$ respectively for the Euler function and the sum of divisors function of the positive integer $n$. There are many open problems concerning the characterization of the positive integers $n$ fulfilling certain congruences involving $\phi(n)$ and $\sigma(n)$. For example, a known open problem due to Lehmer asks if there are any composite integers $n$ such that $n \equiv 1 \bmod \phi(n)$ (see [6]). A different problem due to Subbarao concerns finding composite integers $n$ such that $n \sigma(n) \equiv 2 \bmod \phi(n)$ (see [8]). See also [3, Section B37] for other problems and results of a similar kind.

In this paper, we study a congruence similar to Subbarao's congruence, namely

$$
n \phi(n) \equiv 2 \bmod \sigma(n) .
$$

Congruence (1) was recently proposed and investigated by Díaz (unpublished). It is easy to see that prime numbers $n$ satisfy (1). Diaz showed that the only positive

The first author was supported in part by the Ministry of Science, Education and Sports, Republic of Croatia, grant 037-0372781-2821. The second author was supported in part by Grant SEP-CONACyT 79685.

(C) 2013 Australian Mathematical Publishing Association Inc. 1446-7887/2013 \$16.00 
integers $n$ which are prime powers but not primes that satisfy (1) are $n=8,9$. It was also shown that if $n$ is a composite integer satisfying (1) and if we put

$$
k:=\frac{n \phi(n)-2}{\sigma(n)},
$$

then $n$ can be bounded in terms of $k$. This follows from the minimal order $\phi(n) \gg$ $n / \log \log n$ of the Euler function, as well as the maximal order $\sigma(n) \ll n \log \log n$ of the sum of divisors function, which together imply that

$$
k=\frac{n \phi(n)-2}{\sigma(n)} \gg \frac{n \phi(n)}{\sigma(n)} \gg \frac{n}{(\log \log n)^{2}},
$$

yielding that $n \ll k(\log \log k)^{2}$.

Here, we prove two results about congruence (1). First, we let $\mathcal{P}=\left\{p_{1}, \ldots, p_{k}\right\}$ be a finite set of primes and let $\mathcal{S}_{\mathcal{P}}=\left\{p_{1}^{a_{1}} \cdots p_{k}^{a_{k}}: a_{i} \geq 0\right\}$ be the set of all positive integers whose prime factors belong to $\mathcal{P}$. Our first result is the following theorem.

THEOREM 1. For any finite set of primes $\mathcal{P}$ there are only finitely many positive integers $n \in \mathcal{S}_{\mathcal{P}}$ satisfying congruence (1).

For a positive integer $n$ let $P(n)$ be the largest prime factor of $n$. Theorem 1 has the following immediate corollary.

Corollary 2. $P(n) \rightarrow \infty$ as $n$ goes to infinity through solutions of congruence (1).

The proof of Theorem 1 uses a result of Hernández and Luca [5] whose proof uses Schmidt's subspace theorem and finiteness results about the number of nondegenerate solutions to $\mathcal{S}$-unit equations. As such, it is ineffective. That is, given $\mathcal{P}$, we do not know how to write down a specific upper bound depending on $\mathcal{P}$ on the largest solution $n \in \mathcal{S}_{\mathcal{P}}$ of congruence (1). Our next result is an effective version of Theorem 1 when $\mathcal{P}=\{2,3\}$. Quite likely, our method of proof extends to all sets $\mathcal{P}$ consisting of only two primes but we have not worked out the details of such an extension.

Theorem 3. If $\mathcal{P}=\{2,3\}$, then the only $n \in \mathcal{S}_{\mathcal{P}}$ satisfying congruence (1) are $1,2,3,8$ and 9 .

\section{The proof of Theorem 1}

First we comment on the situation when $n=p^{a}$ for some prime $p$ and exponent $a$ greater than 1. Put

$$
D:=\sigma\left(p^{a}\right)=\frac{p^{a+1}-1}{p-1} .
$$

Then $p^{a+1} \equiv 1 \bmod D$. But also $n \phi(n) \equiv 2 \bmod D$, or $p^{2 a-1}(p-1) \equiv 2 \bmod D$. Hence, $p^{2(a+1)}(p-1) \equiv 2 p^{3} \bmod D$. Using also $p^{a+1} \equiv 1 \bmod D$, we see that $2 p^{3} \equiv p-1 \bmod$ $D$. Thus, $D \mid 2 p^{3}-p+1$. The expression $2 p^{3}-p+1$ is never 0 , so $D \leq 2 p^{3}-p+1$. Thus,

$$
p^{a+1}-1 \leq(p-1)\left(2 p^{3}-p+1\right)
$$


If $a \geq 4$, then $p^{5}-1 \leq p^{a+1}-1 \leq(p-1)\left(2 p^{3}-p+1\right)$, which is impossible. Thus, $a \in\{2,3\}$. If $a=2$, then $p^{2}+p+1 \mid 2 p^{3}-p+1$, which leads to $p^{2}+p+1 \mid p-3$. This is possible only when $p=3$, which gives the solution $n=9$. If $a=3$, then $p^{3}+p^{2}+p+1 \mid 2 p^{3}-p+1$, from which we see that $p^{3}+p^{2}+p+1 \mid 2 p^{2}+3 p+1$. Thus, $p^{3} \leq p^{2}+2 p$, so $p \leq 2$. This leads to the solution $n=8$ to congruence (1).

Now let $\mathcal{P}=\left\{p_{1}, \ldots, p_{k}\right\}$. We assume that $p_{1}<p_{2}<\cdots<p_{k}$. There is no loss of generality in assuming that $\mathcal{P}$ consists of all primes $p \leq p_{k}$, and hence $p_{j}$ is just the $j$ th prime number. Now suppose that $n=p_{i_{1}}^{a_{1}} \cdots p_{i_{s}}^{a_{s}} \in \mathcal{S}_{\mathcal{P}}$ satisfies the congruence (1), where $1 \leq i_{1}<\cdots<i_{s} \leq k$ and the $a_{j}$ are nonnegative for $j=1, \ldots, s$. There is no loss of generality in assuming that $s \geq 2$. Put $u_{j}:=p_{i_{j}}^{a_{j}+1}$ for $j=1, \ldots, s$ and put

$$
v:=n \phi(n) / 2=p_{i_{1}}^{2 a_{1}-1} \cdots p_{s}^{2 a_{s}-1}\left(p_{i_{1}}-1\right) \cdots\left(p_{i_{s}}-1\right) / 2 .
$$

Observe that $u_{j}$ and $v$ are all members of $\mathcal{S}_{\mathcal{P}}$ for $j=1, \ldots, s$. Moreover, $u_{j}$ and $v$ are multiplicatively independent, namely there do not exist integers $x$ and $y$ not both zero such that $u_{j}^{x}=v^{y}$, because $u_{j}$ is a prime power and $v$ has at least two distinct prime factors, namely $p_{i_{1}}$ and $p_{i_{2}}$. Let $j$ be such that $u_{j}=\max \left\{u_{t}: 1 \leq t \leq s\right\}$. We may assume that $a_{j} \geq 3$, otherwise $u_{t} \leq p_{k}^{3}$, for all $i=1, \ldots, s$, so there are only finitely many possibilities for $n$. Then

$$
v<p_{i_{1}}^{2 a_{1}} \cdots p_{i_{s}}^{2 a_{s}}<u_{1}^{2} \cdots u_{s}^{2}<u_{j}^{2 k}
$$

giving that $u_{j}>v^{1 / 2 k}$. Since $\left(u_{j}-1\right) /\left(p_{i_{j}}-1\right)$ divides $2(v-1)$, it follows that

$$
\operatorname{gcd}\left(u_{j}-1, v-1\right) \geq \frac{u_{j}-1}{2\left(p_{i_{j}}-1\right)}>u_{j}^{1 / 2}>v^{1 / 4 k},
$$

where we used the fact that $a_{j} \geq 3$. However, a result of Hernández and Luca from [5] asserts that if $\varepsilon>0$ is fixed, then there are only finitely many pairs of elements $(u, v)$ in $\mathcal{S}_{\mathcal{P}}$ such that

$$
\operatorname{gcd}(u-1, v-1)<\max \{u, v\}^{\varepsilon},
$$

and such that $u$ and $v$ are multiplicatively independent. Note that $u_{j}<v$ for $a_{j} \geq 3$. Since we have already established that $u_{j}$ and $v$ are multiplicatively independent, the above result applied with $\varepsilon:=1 / 4 k$ gives us only finitely many possibilities for $v$. Hence, there are only finitely many possibilities for $n \phi(n)$, and in particular for $n$, which is what we wanted to prove. Theorem 1 is therefore proved.

\section{Proof of Theorem 3}

We assume that $n=2^{a} 3^{b}$, where $a$ and $b$ are positive integers. Let $M:=2^{a+1}-1$ and $N:=\left(3^{b+1}-1\right) / 2$. Then $2^{a+1} \equiv 1 \bmod M$ and $3^{b+1} \equiv 1 \bmod N$. But also $n \phi(n) \equiv$ $2 \bmod M N$, which gives $2^{2 a} 3^{2 b-1} \equiv 2 \bmod M N$. Thus, $2^{2(a+1)} 3^{2(b+1)} \equiv 216 \bmod M N$. Since $2^{a+1} \equiv 1 \bmod M$, we see that $3^{2(b+1)} \equiv 216 \bmod M$. Also, since $3^{b+1} \equiv 1 \bmod N$, 
it follows that $2^{2(a+1)} \equiv 216 \bmod N$. Since $M$ divides $2^{2(a+1)}-1$ and $N$ divides $3^{2(b+1)}-1$, it follows that both $M$ and $N$ divide

$$
2^{2(a+1)}+3^{2(b+1)}-217 .
$$

Let us now show that $a$ and $b$ are both even and that $M$ and $N$ are coprime. Let $D:=\operatorname{gcd}(M, N)$. Then $2^{a+1} \equiv 3^{b+1} \equiv 1 \bmod D$, so $D$ divides $1+1-217=-215=$ $-5 \times 43$. But if 5 divides $M$, then $4 \mid a+1$, so, in particular, $2 \mid a+1$, which implies that $3 \mid M$. This leads to $3 \mid n \phi(n)-2=2^{2 a} 3^{2 b-1}-1$, which is false. Hence, $D$ cannot be a multiple of 5 and $a+1$ is odd, therefore $a$ is even. If 43 divides $M$, then $2^{a+1} \equiv 1 \bmod 43$, which implies again that $a+1$ is even, which is a contradiction. Hence, $M$ and $N$ are coprime and $a$ is even. Let us show that $b$ is also even. If not, then $b+1$ is even, so $3^{b+1}-1$ is a multiple of 8 . Thus, $4|N| 2^{2 a} 3^{2 b-1}-2$, which is impossible. Hence, $b+1$ is odd and therefore both $M$ and $N$ are odd. Since $M N$ divides $2^{2(a+1)}+3^{2(b+1)}-217$ and this last number is even, we deduce that this last number is a multiple of $2 M N=\left(2^{a+1}-1\right)\left(3^{b+1}-1\right)$. Let $x:=2^{a+1}$ and $y:=3^{b+1}$. Then the equation

$$
x^{2}+y^{2}-217=c(x-1)(y-1)
$$

holds, for some positive integer $c$. Since $a$ and $b$ are even, we have the following congruences: $x \equiv 0 \bmod 8, y \equiv 3 \bmod 8, y^{2} \equiv 9 \bmod 16, x \equiv 2 \bmod 3, x^{2} \equiv 1 \bmod 3$, $y \equiv 0 \bmod 3$. Using these congruences, from (2), we conclude that $c \equiv 0 \bmod 8$ and $c \equiv 0 \bmod 3$; that is, $c \equiv 0 \bmod 24$.

We shall next 'diagonalize' equation (2). Namely, let

$$
\begin{aligned}
& X:=c y-c-2 x, \\
& Y:=c y-c-2 y .
\end{aligned}
$$

Then

$$
\begin{aligned}
(c & +2) Y^{2}-(c-2) X^{2}-(-860 c+1736) \\
& =-4(c-2)\left(x^{2}+y^{2}-217-c(x-1)(y-1)\right)=0 .
\end{aligned}
$$

Hence, we arrive at the Pellian equation

$$
(c+2) Y^{2}-(c-2) X^{2}=-860 c+1736 .
$$

From (5), we see that $X / Y$ is a good rational approximation of the irrational number $\sqrt{(c+2) /(c-2)}$. More precisely,

$$
\left|\frac{X}{Y}-\sqrt{\frac{c+2}{c-2}}\right|=\frac{860 c-1736}{(\sqrt{c+2} Y+\sqrt{c-2} X) \sqrt{c-2} Y} \leq \frac{860(c-2)}{\sqrt{c^{2}-4} Y^{2}}<\frac{860}{Y^{2}} .
$$

The rational approximation of the form

$$
\left|\frac{X}{Y}-\sqrt{\frac{c+2}{c-2}}\right|<\frac{860}{Y^{2}}
$$


is not good enough to conclude that $X / Y$ is a convergent of continued fraction expansion of $\sqrt{(c+2) /(c-2)}$, but by Worley's theorem [9, Theorem 1] (see also [1, Theorem 1]), we know that

$$
\frac{X}{Y}=\frac{r p_{k+1} \pm u p_{k}}{r q_{k+1} \pm u q_{k}}
$$

where $k \geq-1$ and $r$ and $u$ are nonnegative integers such that $r u<2 \times 860=1720$. Since $c$ is even, we have the continued fraction expansion

$$
\sqrt{\frac{c+2}{c-2}}=[1, \overline{(c-2) / 2,2}]
$$

(see, for example, [4]). Let $X=d\left(r p_{k+1} \pm u p_{k}\right), Y=d\left(r q_{k+1} \pm u q_{k}\right)$, where $d^{2} r u<$ 1720. Then, by [2, Lemma],

$$
(c+2) Y^{2}-(c-2) X^{2}=d^{2}(-1)^{k}\left(u^{2} t_{k+1}+2 r u s_{k+1}-r^{2} t_{k+2}\right),
$$

where $\left\{s_{k}\right\}_{k \geq-1}$ and $\left\{t_{k}\right\}_{k \geq-1}$ are sequences of integers appearing in the continued fraction algorithm for quadratic irrational $\sqrt{(c+2) /(c-2)}$. From [4], we learn that $s_{k}=c-2, t_{2 k}=c-2, t_{2 k+1}=4$. Let us check whether it is possible that the expression on the right-hand side of (7) is identically equal to the right-hand side of (5); that is, to $-860 c+1736$. If $k$ is even, then $d^{2}\left(\left(4 u^{2}-2 r u+2 r^{2}\right)+c\left(2 r u c-r^{2}\right)\right)$, while if $k$ is odd, then $-d^{2}\left(c\left(u^{2}+2 r u\right)-\left(4 r^{2}+4 r u+2 u^{2}\right)\right)$. Comparing these two expression with $-860 c+1736$, we first see that $d=1$ or $d=2$, and then that in both cases the resulting system of two equations has no integer solutions.

It remains to consider all possible triples of integers $d, r, u$ satisfying $d^{2} r u<1720$, and check whether the corresponding right-hand sides of (7) have nonempty integer intersection with $-860 c+1736$, and lastly compute the corresponding positive integer $c$. There are many such $c$ (the largest is 739586), but only three of them satisfy the condition $c \equiv 0 \bmod 24$. These $c$ are 48, 288 and 23328 .

Let us solve the corresponding three Pellian equations. The equations are:

$$
\begin{aligned}
25 Y^{2}-23 X^{2} & =-19772, \\
145 Y^{2}-143 X^{2} & =-122972, \\
11665 Y^{2}-11663 X^{2} & =-10030172 .
\end{aligned}
$$

Using bounds for the fundamental solutions of Pellian equations (see, for example, [7]), we find that all solutions of equation (8) are given by $\left(X_{0}, X_{1}\right)=$ $(58,192)$ or $(192,58)$ and $X_{k}=48 X_{k-1}-X_{k-2}$ for all $k \geq 2$, and by $\left(Y_{0}, Y_{1}\right)=(48,182)$ or $(182,48)$ and $Y_{k}=48 Y_{k-1}-Y_{k-2}$ for all $k \geq 2$. Assume now that for $X$ and $Y$ defined by (3) and (4) there exists an index $k$ such that $X=X_{k}$ and $Y=Y_{k}$. Then $(X, Y) \equiv$ $(10,0),(0,38),(0,10)$ or $(38,0)$ modulo 48 . But on the other hand, $X \equiv 0 \bmod 16$ and $Y \equiv 0 \bmod 6$, and none of these four pairs satisfies this condition.

Completely analogous arguments apply to the other two equations, since both other $c$ are also divisible by 24 . The fundamental solutions of (9) are $\left(X_{0}, X_{1}\right)=(38,1992)$ 
and $\left(Y_{0}, Y_{1}\right)=(24,1978)$, and we see that $(X, Y) \equiv(14,0),(0,10),(0,14)$ or $(10,0)$ modulo 24, while the fundamental solutions of $(10)$ are $\left(X_{0}, X_{1}\right)=(218,23112)$ and $\left(Y_{0}, Y_{1}\right)=(216,23110)$, and so $(X, Y) \equiv(2,0),(0,22),(0,2)$ or $(22,0)$ modulo 24 . In both cases, none of the pairs modulo 24 satisfies the conditions $X \equiv 0 \bmod 16$ and $Y \equiv 0 \bmod 6$. This completes the proof of Theorem 3 .

\title{
Acknowledgement
}

This work was done while the first author visited the Mathematical Institute of the Universidad Nacional Autónoma de México in Morelia in December 2010. He thanks the people of this institution for their hospitality and support.

\section{References}

[1] A. Dujella, 'Continued fractions and RSA with small secret exponents', Tatra Mt. Math. Publ. 29 (2004), 101-112.

[2] A. Dujella and B. Jadrijević, 'A family of quartic Thue inequalities', Acta Arith. 111 (2004), 61-76.

[3] R. Guy, Unsolved Problems in Number Theory, 2nd edn, Problem Books in Mathematics (Springer, New York, 1994).

[4] B. He, B. Jadrijević and A. Togbé, 'Solutions of a class of quartic Thue inequalities', Glas. Mat. Ser. III 44 (2009), 309-321.

[5] S. H. Hernández and F. Luca, 'On the largest prime factor of $(a b+1)(a c+1)(b c+1)$ ', Bol. Soc. Mat. Mexicana 9 (2003), 235-244.

[6] D. H. Lehmer, 'On Euler's totient function', Bull. Amer. Math. Soc. 38 (1932), 745-751.

[7] T. Nagell, Introduction to Number Theory (Almqvist, Stockholm; Wiley, New York, 1951).

[8] M. V. Subbarao, 'On two congruences for primality', Pacific J. Math. 52 (1974), 261-268.

[9] R. T. Worley, 'Estimating $|\alpha-p / q|$ ', J. Aust. Math. Soc. Ser. A 31 (1981), 202-206.

\author{
ANDREJ DUJELLA, Department of Mathematics, University of Zagreb, \\ Bijenička cesta 30, 10000 Zagreb, Croatia \\ e-mail: duje@math.hr
}

FLORIAN LUCA, Fundación Marcos Moshinsky, Instituto de Ciencias Nucleares UNAM, Circuito Exterior, C.U., Apdo. Postal 70-543, Mexico D.F. 04510, Mexico

e-mail: fluca@matmor.unam.mx 